Audiology

Neurotology
Audiol Neurotol 2012;17:373-380

DOI: $\underline{10.1159 / 000341818}$
Received: March 13, 2012

Accepted after revision: July 9, 2012

Published online: August 15, 2012

\title{
The Clinical Significance of Preoperative Brain Magnetic Resonance Imaging in Pediatric Cochlear Implant Recipients
}

\author{
Il Joon Moon ${ }^{\mathrm{a}}$ Eun Yeon Kim ${ }^{\mathrm{a}}$ Ga-Young Park ${ }^{\mathrm{a}}$ Min Seok Jang ${ }^{\mathrm{a}}$ Ji Hye Kim ${ }^{\mathrm{b}}$ \\ Jeehun Lee ${ }^{c}$ Won-Ho Chung ${ }^{a}$ Yang-Sun Cho ${ }^{a}$ Sung Hwa Hong ${ }^{a}$ \\ ${ }^{a}$ Department of Otorhinolaryngology - Head and Neck Surgery, ${ }^{b}$ Department of Radiology and Center for \\ Imaging Science, and ' Department of Pediatrics, Samsung Medical Center, Sungkyunkwan University School of \\ Medicine, Seoul, Korea
}

\section{Key Words \\ Cochlear implants - Magnetic resonance imaging $\cdot$ Brain damage, chronic - Auditory perception $\cdot$ Prognosis $\cdot$ Child}

\begin{abstract}
Although central nervous system abnormalities are incidentally detected in preoperative brain magnetic resonance imaging (MRI) studies in pediatric cochlear implant (Cl) candidates, the clinical significance of the abnormalities remains unclear. We aimed to assess post-implantation auditory and speech performance in patients with brain lesions seen on MRI. Pediatric $\mathrm{Cl}$ recipients $(n=177)$ who underwent preoperative MRI scans of the brain between January 2002 and June 2009 were included in this study. Patients with brain lesions on MRI were reviewed and categorized into the following groups: brain parenchymal lesions (focal vs. diffuse), ventriculomegaly, and extra-axial lesion. The main communication mode as well as progress in auditory perception and speech production were evaluated preoperatively and at 3 , 6,12 , and 24 months postoperatively. Performance in patients with brain lesions was compared with the age- and sex-matched control group. Various brain lesions were found in 27 out of 177 patients. Children with brain lesions who re-
\end{abstract}

ceived $\mathrm{Cls}$ showed gradual progress in auditory and speech outcomes for 2 years, though performance was reduced compared with the control group. In addition, there was a significant difference in the main communication mode between the two groups at 2 years following cochlear implantation. This difference was especially significant in patients with diffuse brain parenchymal lesions after further stratification of the brain lesion group. Preoperative brain MRI may have a role in improving the prediction of adverse outcomes in pediatric $\mathrm{Cl}$ recipients. In particular, children with diffuse brain parenchymal lesions should be counseled regarding the poor prognosis preoperatively, and followed up with special attention.

Copyright $\odot 2012$ S. Karger AG, Basel

\section{Introduction}

Preoperative diagnostic protocols for pediatric cochlear implant (CI) candidates presenting with profound sensorineural hearing loss vary among physicians and clinics, but radiographic imaging gives valuable information in these patients. Historically, high-resolution CT has been the primary imaging modality in the initial workup

\section{KARGER}

Fax +4161306 1234

E-Mail karger@karger.ch

www.karger.com
(C) 2012 S. Karger AG, Basel

$1420-3030 / 12 / 0176-0373 \$ 38.00 / 0$

Accessible online at:

www.karger.com/aud
Sung Hwa Hong, MD, PhD

Department of Otorhinolaryngology - Head and Neck Surgery

Samsung Medical Center, Sungkyunkwan University School of Medicine

50 Irwon-dong, Gangnam-gu, Seoul 135-710 (Korea)

Tel. +82 234103579 , E-Mail hongsh@skku.edu 
of patients with profound hearing loss [Antonelli et al., 1999; Hone and Smith, 2002]. However, high-resolution CT is not sufficient to evaluate the soft tissue structures of the inner ear, such as the membranous labyrinth and the vestibulocochlear nerve. Magnetic resonance imaging (MRI) can directly assess these soft tissue structures responsible for hearing. Therefore, high-resolution brain (or internal auditory canal) MRI has been an important tool in investigating the etiology of deafness and in the selection process for cochlear implantation (CI) [Gleeson et al., 2003; Lapointe et al., 2006; Kutz et al., 2011]. Additionally, brain MRI allows physicians to detect abnormalities of the central nervous system (CNS) that may adversely affect neurological development as well as cognitive function [Bouhadiba et al., 2000; Hart et al., 2008]. For example, white matter lesions in the brain can be easily detected as high-intensity lesions on proton density and T2-weighted MRI scans [Inaba et al., 2011]. Therefore, in many institutions, preoperative brain MRI is routinely performed before CI, not only to evaluate the status of the inner ear and cochlear nerve, but also to screen for CNS disorders.

In these imaging studies, physicians have usually focused on the inner ear structures and vestibulocochlear nerve. However, structural CNS abnormalities may be incidentally found in some pediatric CI candidates. These brain lesions include various degrees of white and gray matter lesions, ventriculomegaly, and extra-axial lesions such as arachnoid cysts. In particular, cerebral lesions such as white matter abnormalities are known as useful markers for an elevated risk of psychomotor delay, cognitive delay, cerebral palsy, and neurosensory impairment [Inder et al., 2005; Woodward et al., 2005, 2006; Inzitari et al., 2007].

However, the relationships between the severity of clinical presentation and the severity of MRI findings in CNS abnormalities are not fully understood. A substantial proportion of children with brain lesions are free of severe impairment, especially at young ages [Woodward et al., 2006]. Therefore, if some pediatric CI candidates with structural CNS abnormalities do not have severe neurologic symptoms, these patients can be easily overlooked during the rehabilitation process after CI. Children who show structural CNS abnormalities may have poor outcomes after CI, but the clinical significance of brain lesions on imaging has remained unclear so far.

The aim of this study was to evaluate the impact of CNS abnormalities seen on imaging on post-implantation auditory and speech performance in young deaf children.
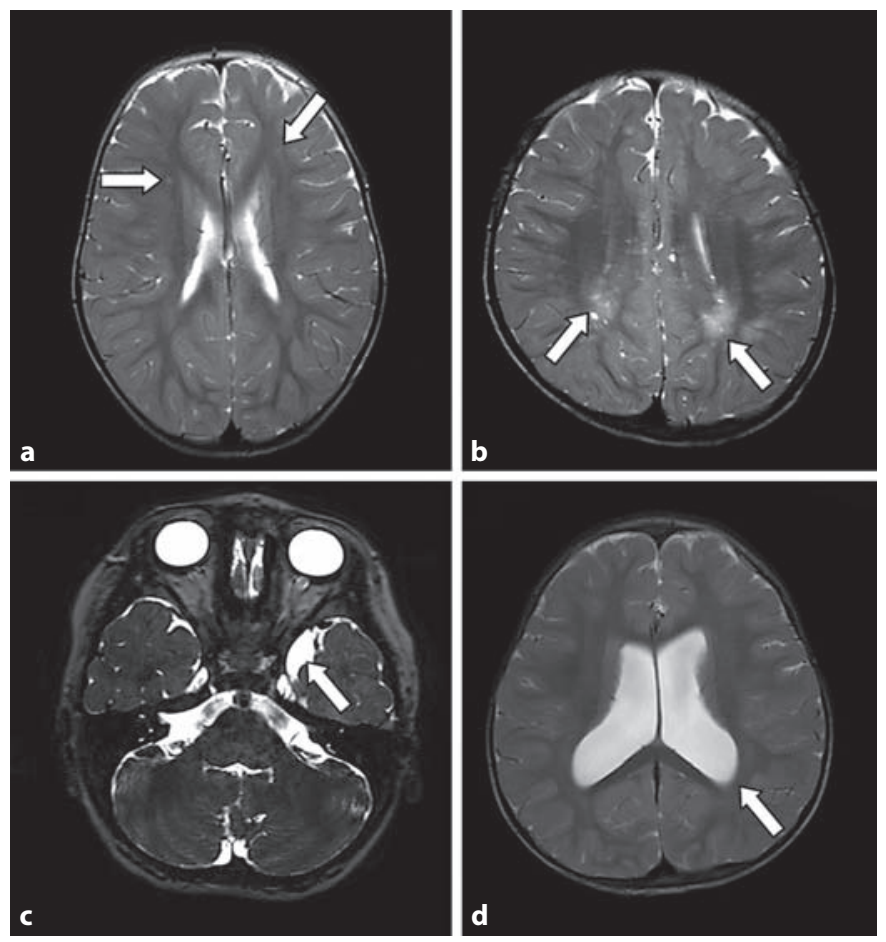

Fig. 1. Abnormal brain MRI according to classification. Focal parenchymal high signal lesions in the periventricular white matter (focal parenchymal lesion) (a), multiple and diffuse deep white matter high signal lesions (diffuse parenchymal lesion) (b), arachnoid cyst in the left middle fossa (extra-axial lesion) (c), and ventriculomegaly (d).

\section{Patients and Methods}

We retrospectively reviewed the medical charts of 177 consecutive prelingually deaf children ( 98 males and 79 females, $<12$ years of age) who underwent CI at the Samsung Medical Center between January 2002 and June 2009. All of the patients underwent preoperative MRI scans of the brain and were followed for more than 2 years.

Preoperative MR images of these 177 patients were analyzed by two pediatric radiologists, and brain lesions were found in 27 (14 males and 13 females). These lesions were divided into the following four groups: (1) focal cerebral white matter lesions $(n=7)$; (2) diffuse cerebral white matter lesions $(n=12)$; (3) extra-axial lesions such as arachnoid cysts $(\mathrm{n}=3)$, and (4) ventriculomegaly $(n=5)$ (fig. 1). If the white matter lesion was $<10 \%$ of the whole white matter, the lesion was categorized as a 'focal lesion'. If the white matter lesion was found to be $>10 \%$ of the whole brain, the lesion was classified as a 'diffuse lesion'.

After review of MR images, patients with brain lesions (brain lesion group) were age- and sex-matched with prelingually deaf children without brain lesions (control group) for comparison of auditory and speech outcomes. In addition, all patients with brain lesions were evaluated by a pediatric neurologist to examine neurodevelopmental status as well as to investigate the presence of cerebral palsy. 
Table 1. Demographic data of patients including associated disabilities

\begin{tabular}{llll}
\hline & Brain lesion group & Control group & p value* $^{*}$ \\
\hline Patients, n & 27 & 27 & 1.000 \\
Age at implantation, months & $43.6 \pm 31.3$ & $42.6 \pm 29.3$ & 0.906 \\
Etiology of deafness & all congenital & all congenital & 1.000 \\
Associated cerebral palsy, n & 9 & 0 & 0.002 \\
Associated developmental delay, n & 4 & 0 & 0.111 \\
Associated inner ear anomaly, n & 9 & 5 & 0.352 \\
\hline
\end{tabular}

* By Fisher's exact test.

Table 2. Distribution of additional disabilities in the brain lesion group

\begin{tabular}{llll}
\hline & $\begin{array}{l}\text { Cerebral } \\
\text { palsy }\end{array}$ & $\begin{array}{l}\text { Neuro- } \\
\text { developmental } \\
\text { delay }\end{array}$ & $\begin{array}{l}\text { Inner } \\
\text { ear } \\
\text { anomaly }\end{array}$ \\
\hline Focal parenchymal lesion $(\mathrm{n}=7)$ & 3 & 0 & 0 \\
Diffuse parenchymal lesion $(\mathrm{n}=12)$ & $5^{*}$ & $4^{*}$ & 6 \\
Extra-axial lesion $(\mathrm{n}=3)$ & 0 & 0 & 0 \\
Ventriculomegaly $(\mathrm{n}=5)$ & 1 & 0 & 3 \\
\hline
\end{tabular}

* One patient in the diffuse lesion group had both cerebral palsy and neurodevelopmental delay.

Patients who had intra- or postoperative complications and those without sufficient follow-up ( $<2$ years) were excluded from this study. The ages of the brain lesion and control groups ranged from 12 to 133 months with mean ages of 43.6 and 42.6 months, respectively (table 1). Distributions of additional disabilities, such as cerebral palsy or neurodevelopmental delay, according to the classification of brain lesions, are summarized in table 2.

Auditory perception was measured using Categories of Auditory Performance (CAP), and speech ability was measured using the Korean version of Ling's stages (K-Ling) [Moon et al., 2011] in both groups. Using the K-Ling, the level of phonologic and phonetic development was assessed at baseline (preoperative) and at $3,6,12$, and 24 months after the initial mapping.

In addition, to establish the children's main communication mode, an experienced speech therapist (E.Y.K.) assessed patient ability to communicate in daily situations during rehabilitation using the K-Ling. If a patient's phonologic and phonetic levels were $>2$, the patient's main communication mode was classified as oral communication. The patients who achieved level 3-7 in the K-Ling could use speech as a main communication mode and use correct vowel sounds as well as a more complex phonetic inventory. If both the phonologic and phonetic levels of a patient were 0 , then the patient was classified as a non-verbal communicator. Others with phonetic and phonologic level 1-2 were classified into the vocalization only group. These patients could control their phonation and produce consonant-vowel syllables including vow- el nuclei. Hence, they could use various types of vocalization as a means of communication.

In order to analyze the repeated measures data, a generalized estimating equation was used in this study. Previously known confounding factors, such as preoperative communication mode, additional disabilities such as mental retardation or cerebral palsy, auditory cortex involvement, and inner ear anomalies were adjusted for by including them in the models. Fisher's exact test and independent $t$ test were used for group analysis. The statistical analyses were performed using Predictive Analytic Soft Ware (PASW Statistics 18, IBM Corporation, Armonk, N.Y., USA). This study was approved by the Institutional Review Board of the Samsung Medical Center (IRB No. 2010-09-054).

\section{Results}

There were no significant differences in age at implantation $(\mathrm{p}=0.906)$ or etiology of deafness $(\mathrm{p}=1.000)$ between children with brain lesions and those without. The mean warble tone threshold in the brain lesion group was $29.89( \pm 4.10) \mathrm{dB}$ and was $27.60( \pm 3.46) \mathrm{dB}$ in the control group $(\mathrm{p}=0.035)$.

At preoperative evaluation, the difference in communication mode between the brain lesion and control groups was not statistically significant $(p=0.374)$. Nonverbal communication was mainly used in $20(74.1 \%)$ of 27 patients in the brain lesion group and 15 (55.6\%) of 27 patients in the control group (fig. $2 \mathrm{a}, \mathrm{b}$ ). However, at 2 years following implantation, non-verbal communication or vocalization only were observed in 10 patients (37\%) of the brain lesion group, while all of the control group communicated orally (fig. $2 c, d$ ); this difference was statistically significant $(\mathrm{p}<0.001)$.

We further analyzed this result after stratification, and when the brain lesion group was divided into diffuse parenchymal lesions and other lesion groups, statistical significance was observed. Before CI, communication 
Fig. 2. Preoperative (preop) and postoperative (2 years) communication modes of brain lesion and control groups. Most patients in both the brain lesion and control groups used non-verbal communication preoperatively $(\mathbf{a}, \mathbf{b})$, and the difference between the two groups was not statistically significant $(\mathrm{p}=0.374)$. However, at 2 years after implantation, there was a significant difference in communication mode between the two groups $(\mathrm{p}<0.001)$. All of the control group patients used oral communication, while $37 \%$ of the brain lesion group could not communicate orally (c, d).

Fig. 3. Preoperative (preop) and postoperative (2 years) communication modes of diffuse parenchymal lesion and other lesion groups. Most patients in both the diffuse parenchymal lesion and other lesion groups used non-verbal communication preoperatively $(\mathbf{a}, \mathbf{b})$, and the difference between the two groups was not statistically significant $(\mathrm{p}=0.248)$. However, at 2 years after implantation, there was a significant difference in communication mode between the two groups $(\mathrm{p}=0.027)$. Almost all patients in the other lesion groups used oral communication, while $66.7 \%$ of the diffuse parenchymal lesion group could not communicate orally $(\mathbf{c}, \mathbf{d})$.
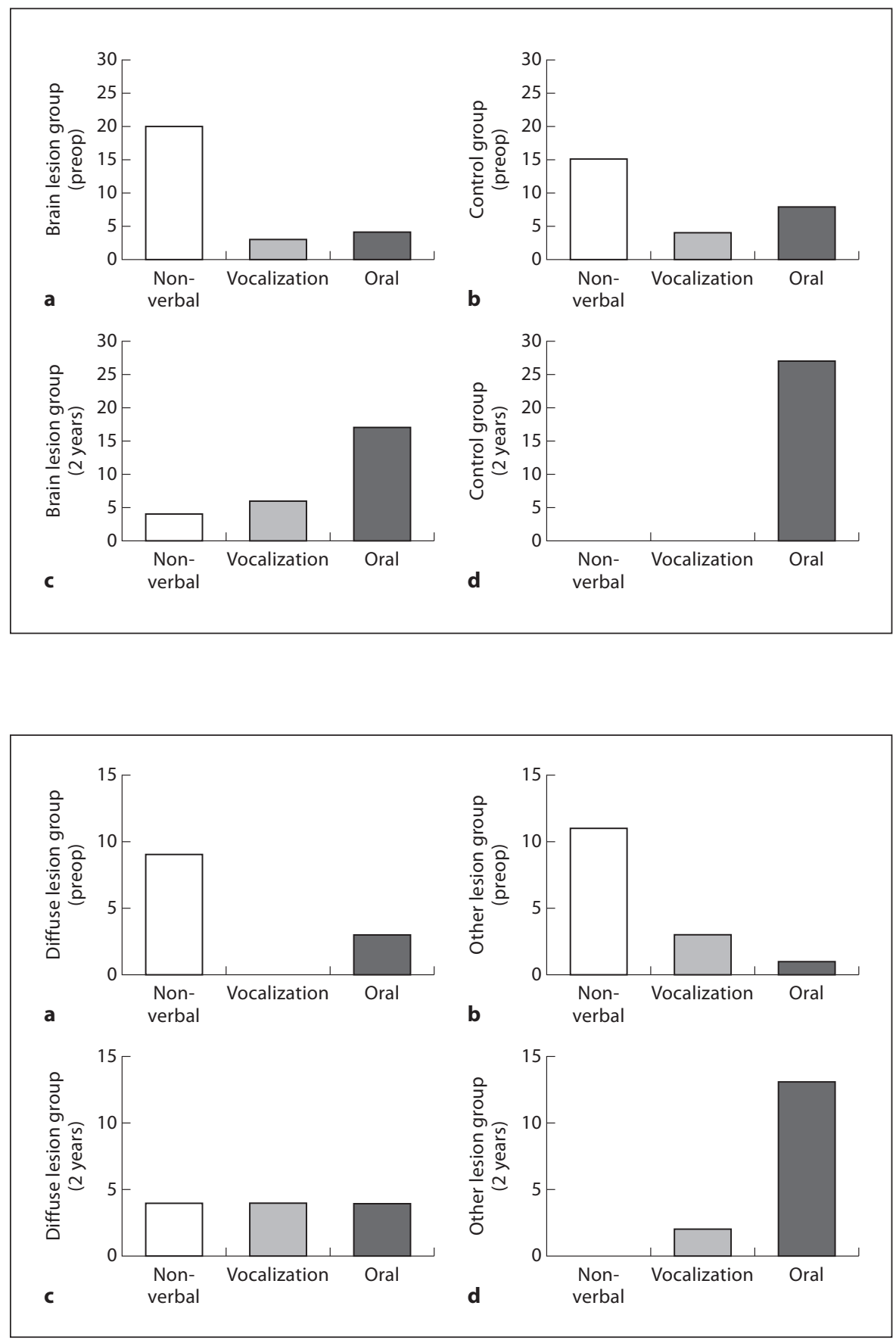

mode was not different between these two groups (fig. 3a, $\mathrm{b} ; \mathrm{p}=0.248)$. However, there was a striking difference in communication mode between these two groups at 2 years following CI $(\mathrm{p}=0.027)$. Almost all patients, 13 $(86.7 \%)$ out of 15 children, in the other brain lesion group used oral communication, while two thirds of children with diffuse parenchymal lesions could not communi- cate orally at that time. All non-verbal communicators were in the diffuse parenchymal lesion group.

To analyze the repeated-measures data, a generalized estimation equation model was used, and a summary of the auditory and speech outcomes for each group is shown in figure 4 . Figure 4 a shows the changes in CAP score between the control and brain lesion groups. After 
Fig. 4. Preoperative (pre) and postoperative CAP scores and K-Ling phonemic and phonologic levels. CAP scores (a) as well as phonemic (c) and phonologic levels (e) of the K-Ling in both the brain lesion and control groups improved continuously up to 24 months, although there was a significant difference between the two groups $(\mathrm{p}<0.05)$. These scores and levels improved to a limited extent in the diffuse parenchymal lesion group for 2 years after implantation, while they improved consistently in other lesion groups $(\mathbf{b}, \mathbf{d}, \mathbf{f})$; the differences between the two groups were statistically significant $(\mathrm{p}<0.05)$. Error bars indicate standard deviations of averages.

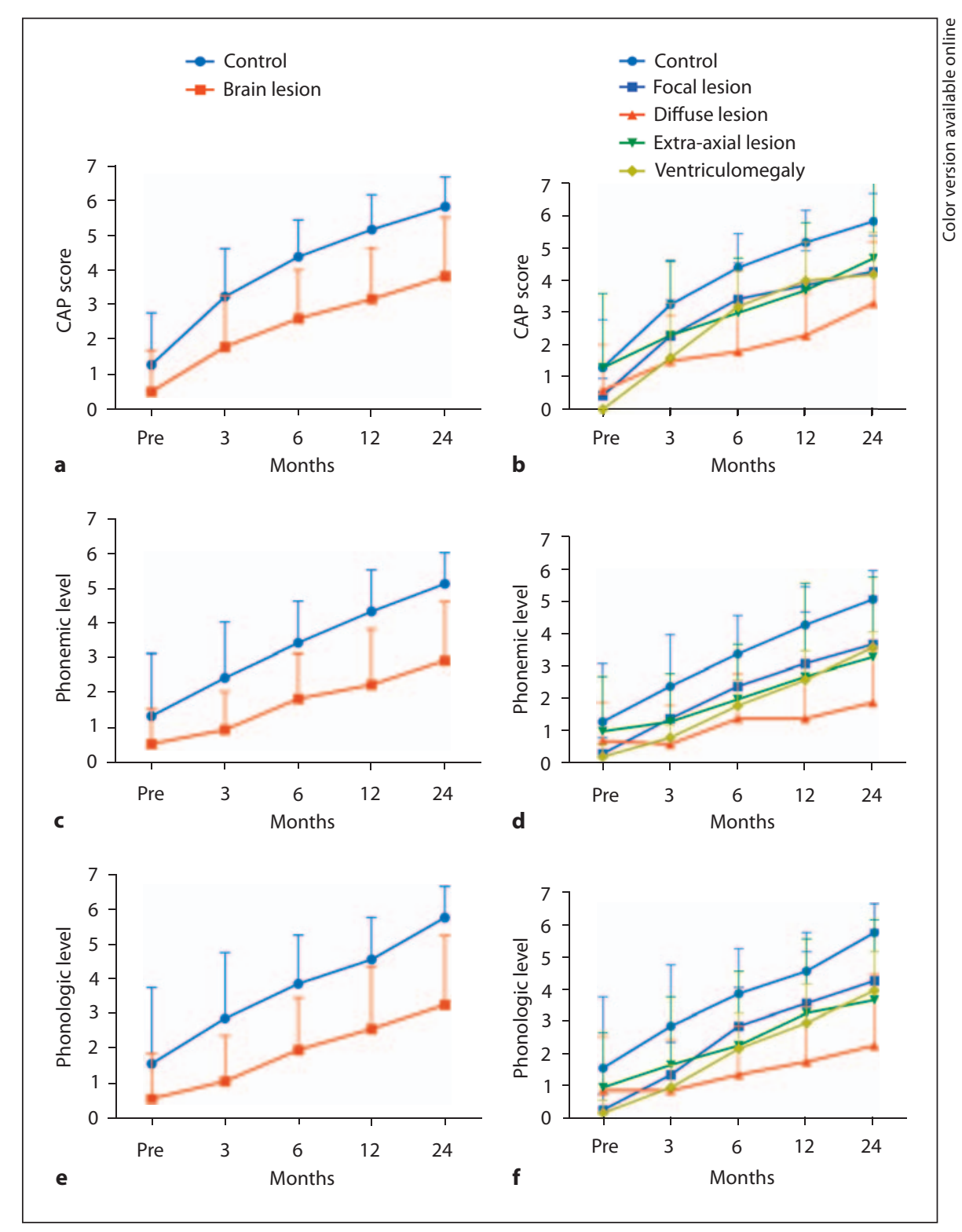

implantation, the CAP scores of the control group improved considerably for 24 months $(\mathrm{p}<0.001)$, and those of children with brain lesions also improved significantly $(\mathrm{p}<0.001)$. However, the control group showed better results than the brain lesion group, and this difference was significant $(p=0.009)$. Figure $4 \mathrm{~b}$ shows the CAP score changes over 24 months after CI according to the various groups. The control group showed the best score, and the performance of the diffuse lesion group improved to a limited extent compared with that of the control group; the difference was statistically significant ( $\mathrm{p}=$ 0.016).
The pattern of changes in the K-Ling's phonemic level was similar to that in the CAP score. The control and brain lesion groups showed steady improvement for 24 months ( $p<0.001$ and $p<0.001$, respectively), and the difference between these two groups was statistically significant ( $\mathrm{p}=0.015$; fig. $4 \mathrm{c}$ ). Like the CAP score, the phonemic level of the diffuse lesion group did not improve significantly compared with the other lesion groups and the control group ( $\mathrm{p}=0.004$; fig. $4 \mathrm{~d})$.

In addition, the phonologic level of the K-Ling showed similar results to the CAP score and the phonemic level. Figure $4 \mathrm{e}$ also shows that even in the brain lesion group, 
the phonologic level increased with time after CI ( $p<$ 0.001 ). However, the performance of the brain lesion group was lower than that of the control group $(\mathrm{p}=0.011)$. Likewise, the diffuse lesion group consistently showed the worst results among all groups $(\mathrm{p}=0.018)$.

\section{Discussion}

Premature birth, low birth weight, and intensive care $>7$ days are risk factors associated with hearing loss in infants [Bielecki et al., 2011]. In addition, sensorineural hearing loss is associated with a variety of additional disabilities in about $30 \%$ of subjects [Holden-Pitt and Albertorio, 1998; Fortnum et al., 2002]. In addition, cytomegalovirus infection, rubella, syphilis, toxoplasmosis, or other viral infections during the prenatal period can lead to profound hearing loss as well as brain lesions [Kral and O’Donoghue, 2010]. For example, abnormal white matter signals are typically related to either pre-perinatal problems, such as hypoxic brain damage, or intrauterine infections [Chilosi et al., 2010]. Hence, there is an increased incidence of brain abnormalities in children with hearing loss.

Brain lesions, such as white matter abnormalities, are useful markers for risk of psychomotor delay, cognitive delay, cerebral palsy, and neurosensory impairment [Inder et al., 2005; Woodward et al., 2005, 2006; Inzitari et al., 2007]. All of these conditions are negative prognostic factors for speech and language development following CI [Holt and Kirk, 2005; Edwards et al., 2006; Wiley et al., 2008; Lee et al., 2010; Steven et al., 2011]. Therefore, we hypothesize that children with brain lesions may have poor outcomes after CI due to impairment of higher cognitive function and perceptual processing, even though they may not display severe clinical symptoms at the time of implantation.

These additional disabilities in a child with profound hearing loss may have important consequences when assessing and choosing a therapeutic treatment, particularly when considering CI. There has been little universal agreement on the potential benefits of CI for children with additional CNS disabilities. Therefore, CI in children with profound sensorineural hearing loss and associated CNS abnormalities is an emerging issue in selecting an appropriate candidate who would gain maximal benefit from implantation.

A substantial proportion of children with brain lesions on imaging in our sample were free of severe impairment at a young age. In our study, 15 (55.6\%) out of 27 patients with brain lesions did not show significant neurodevelopmental impairment at implantation. Therefore, this finding underscores the necessity of performing MRI before CI.

About $15 \%$ of pediatric CI recipients in this study had a variety of abnormal findings in preoperative brain $\mathrm{MR}$ images. Although a significant proportion of children had brain lesions, the patients with brain lesions showed gradual progress in auditory perception and speech production for 2 years following CI. It was important, however, to ensure that the control group, those without brain lesions, demonstrated better performance than the brain lesion group. After further classification of the brain lesion group, the abilities of auditory perception, speech production, and oral communication improved to only a very limited extent in children with diffuse parenchymal lesions.

These interesting findings are in accordance with the results of a recent study, which demonstrated that certain CNS abnormalities may be associated with a poor rehabilitation process and outcome following CI [Hong et al., 2010]. However, the previous study was a descriptive study, and the authors did not compare the outcomes with those of patients without brain lesions.

In our study, the post-CI warble tone thresholds in the brain lesion and control groups were 29.9 and $27.6 \mathrm{~dB}$, respectively. Although this was a statistically significant difference, the threshold in the brain lesion group was sufficient to hear sound. Therefore, the decreased performance in the brain lesion group may be due to the impairment of auditory processing after sound input.

White matter is a tract through which information passes between different areas of gray matter within the CNS [Alix and Domingues, 2011]. White matter lesions on brain MRI have been known to be associated with functional decline as well as neurodevelopmental impairment, such as severe psychomotor delay, cerebral palsy, and impaired working memory [Woodward et al., 2005, 2006; Inzitari et al., 2007; Inaba et al., 2011], because cognitive function relies on processing speed and widely distributed neural networks [Gunning-Dixon and Raz, 2000].

Therefore, patients with diffuse parenchymal lesions may have decreased abilities in processing speed and connection of neural networks that negatively impact specific cognitive domains. In our study, only the diffuse parenchymal lesion group showed a significant delay in performance following CI compared to the control group. Moreover, all non-verbal communicators 2 years after CI were included in the diffuse lesion group. That indicates 
that patients with hearing loss who have diffuse parenchymal abnormalities may have difficulties with perceptual processing of auditory information in the brain.

However, patients with focal parenchymal lesions, ventriculomegaly only, and arachnoid cysts did not show significant differences in auditory and speech outcomes compared with the control group. If the extent of a white matter lesion is not wide enough to interrupt auditory perception processing, then it may not be a significant predictor of poor outcome. In addition, the existence of ventriculomegaly only or arachnoid cysts may not affect perceptual processing and higher cognitive function at all.

In this study, 9 patients in the brain lesion group had a variety of inner ear anomalies (table 2 ). Inner ear anomalies in the diffuse brain parenchymal lesion group were 2 incomplete partition type II, 2 vestibular hypoplasias, 1 enlarged vestibular aqueducts, and 1 internal auditory canal narrowing. Inner ear anomalies in the ventriculomegaly group were 1 incomplete partition type II, 1 vestibular hypoplasia, and 1 enlarged vestibular aqueducts. In order to remove the effect of previously known confounding factors, such as inner ear anomalies, the factors were adjusted for by including them in the models.

A limitation of this study was that we did not perform cognitive function tests examining working memory or intellectual ability in the patients with brain lesions. Hence, the exact relationship between brain lesions and cognitive impairment cannot be elucidated, and further studies regarding this issue are needed to address our hypothesis. Nonetheless, we demonstrated the potential of
MRI performed before CI to improve the prediction of adverse auditory, speech, and language outcomes in children. Our findings suggest that the identification of brain abnormalities with the use of MRI provides valuable information during CI work-up and improves the identification of CI recipients who are at higher risk of poor outcomes.

In conclusion, children with brain lesions obtained demonstrable benefits in communication mode, auditory perception, and speech production from CI. This means that brain lesions themselves should not be considered contraindications for CI. However, children with diffuse parenchymal lesions should be counseled with the parents regarding the poor prognosis preoperatively, and followed up more frequently than normal peers without those lesions.

\section{Acknowledgement}

This work was supported by grants from the Strategic Technology Development Program of Ministry of Knowledge Economy (grant No. 10031764) and Seoul R\&D Program (SS100022), Republic of Korea.

\section{Disclosure Statement}

The authors report no conflicts of interest concerning the materials or methods used in this study or the findings specified in this paper.

\section{References}

Alix JJ, Domingues AM: White matter synapses: form, function, and dysfunction. Neurology 2011;76:397-404.

-Antonelli PJ, Varela AE, Mancuso AA: Diagnostic yield of high-resolution computed tomography for pediatric sensorineural hearing loss. Laryngoscope 1999;109:1642-1647.

- Bielecki I, Horbulewicz A, Wolan T: Risk factors associated with hearing loss in infants: an analysis of 5282 referred neonates. Int J Pediatr Otorhinolaryngol 2011;75:925-930.

- Bouhadiba Z, Dacher J, Monroc M, Vanhulle C, Menard JF, Kalifa G: [MRI of the brain in the evaluation of children with developmental delay]. J Radiol 2000;81:870-873.
Chilosi AM, Comparini A, Scusa MF, Berrettini S, Forli F, Battini R, Cipriani P, Cioni G: Neurodevelopmental disorders in children with severe to profound sensorineural hearing loss: a clinical study. Dev Med Child Neurol 2010;52:856-862.

Edwards LC, Frost R, Witham F: Developmental delay and outcomes in paediatric cochlear implantation: implications for candidacy. Int J Pediatr Otorhinolaryngol 2006;70: 1593-1600.

Fortnum HM, Marshall DH, Summerfield AQ: Epidemiology of the UK population of hearing-impaired children, including characteristics of those with and without cochlear implants - audiology, aetiology, comorbidity and affluence. Int $\mathrm{J}$ Audiol 2002;41:170-179.
-Gleeson TG, Lacy PD, Bresnihan M, Gaffney R, Brennan P, Viani L: High resolution computed tomography and magnetic resonance imaging in the pre-operative assessment of cochlear implant patients. J Laryngol Otol 2003; 117:692-695.

Gunning-Dixon FM, Raz N: The cognitive correlates of white matter abnormalities in normal aging: a quantitative review. Neuropsychology 2000;14:224-232.

Hart AR, Whitby EW, Griffiths PD, Smith MF: Magnetic resonance imaging and developmental outcome following preterm birth: review of current evidence. Dev Med Child Neurol 2008;50:655-663. 
Holden-Pitt L, Albertorio J: Thirty years of the annual survey of deaf and hard-of-hearing children \& youth: a glance over the decades. Am Ann Deaf 1998;143:72-76.

Holt RF, Kirk KI: Speech and language development in cognitively delayed children with cochlear implants. Ear Hear 2005;26:132148 .

-Hone SW, Smith RJ: Medical evaluation of pediatric hearing loss. Laboratory, radiographic, and genetic testing. Otolaryngol Clin North Am 2002;35:751-764.

Hong P, Jurkowski ZC, Carvalho DS: Preoperative cerebral magnetic resonance imaging and white matter changes in pediatric cochlear implant recipients. Int J Pediatr Otorhinolaryngol 2010;74:658-660.

-Inaba M, White L, Bell C, Chen R, Petrovitch H, Launer L, Abbott RD, Ross GW, Masaki K: White matter lesions on brain magnetic resonance imaging scan and 5-year cognitive decline: the Honolulu-Asia aging study. J Am Geriatr Soc 2011;59:1484-1489.

Inder TE, Warfield SK, Wang H, Huppi PS, Volpe JJ: Abnormal cerebral structure is present at term in premature infants. Pediatrics 2005; $115: 286-294$.
Inzitari D, Simoni M, Pracucci G, Poggesi A, Basile AM, Chabriat H, Erkinjuntti T, Fazekas F, Ferro JM, Hennerici M, Langhorne P, O’Brien J, Barkhof F, Visser MC, Wahlund LO, Waldemar G, Wallin A, Pantoni L: Risk of rapid global functional decline in elderly patients with severe cerebral age-related white matter changes: the LADIS study. Arch Intern Med 2007;167:81-88.

Kral A, O'Donoghue GM: Profound deafness in childhood. N Engl J Med 2010;363:14381450 .

Kutz JW Jr, Lee KH, Isaacson B, Booth TN, Sweeney MH, Roland PS: Cochlear implantation in children with cochlear nerve absence or deficiency. Otol Neurotol 2011;32:956-961.

Lapointe A, Viamonte C, Morriss MC, Manolidis S: Central nervous system findings by magnetic resonance in children with profound sensorineural hearing loss. Int J Pediatr Otorhinolaryngol 2006;70:863-868.

Lee YM, Kim LS, Jeong SW, Kim JS, Chung SH: Performance of children with mental retardation after cochlear implantation: speech perception, speech intelligibility, and language development. Acta Otolaryngol 2010; 130:924-934.
Moon IJ, Kim EY, Chu H, Chung WH, Cho YS, Hong SH: A new measurement tool for speech development based on Ling's stages of speech acquisition in pediatric cochlear implant recipients. Int J Pediatr Otorhinolaryngol 2011;75:495-499.

Steven RA, Green KM, Broomfield SJ, Henderson LA, Ramsden RT, Bruce IA: Cochlear implantation in children with cerebral palsy. Int J Pediatr Otorhinolaryngol 2011;75: 1427-1430.

-Wiley S, Meinzen-Derr J, Choo D: Auditory skills development among children with developmental delays and cochlear implants. Ann Otol Rhinol Laryngol 2008;117:711718

Woodward LJ, Anderson PJ, Austin NC, Howard $\mathrm{K}$, Inder TE: Neonatal MRI to predict neurodevelopmental outcomes in preterm infants. N Engl J Med 2006;355:685-694.

-Woodward LJ, Edgin JO, Thompson D, Inder TE: Object working memory deficits predicted by early brain injury and development in the preterm infant. Brain 2005;128:2578-2587. 